\title{
Does the Overhead Squat Assessment is needed as a Movement Screening Method in Health Care Practice
}

\author{
Senthil P* \\ Associate Professor, School of Physiotherapy, VISTAS, India \\ *Corresponding author: Senthil Purushothaman, Associate Professor, School of \\ Physiotherapy, Vels Institute of Science, Technology and Advanced Studies (VISTAS), \\ Chennai, India, Email: senthilp101010@gmail.com
}

\section{Mini Review \\ Volume 3 Issue 2}

Received Date: March 25, 2019

Published Date: April 12, 2019

DOI: $10.23880 /$ jobd- 16000177

\section{Mini Review}

Globally, the incidence of musculoskeletal dysfunction and its related movement impairment have been extremely raised in varying population. If this movement impairment is not identified earlier with proper musculoskeletal screening, it will leads to recurrent complaint of musculoskeletal system and thereby muscle fatigue and lesser productivity. There are many movement screening methods which has been practiced in the health sector. But the overhead squat assessment is the simple and time-effective key assessments for an indication of gross movement quality. The National Academy of Sports Medicine (NASM) also prefer to focus on what movement compensation occurs at each joint with possible over-active and under-active muscles that could be contributing to the dysfunction and provides a score ranging from 0-3 (3 = performed with perfect form, $2=$ performed with compensation, 1 = performed poorly and $0=$ unable to perform the assessment due to pain) [1]. Using this numerical rating score for the initial assessment by the researchers can be useful to compare with the re-assessment. Ultimately, NASM's method delves into the possible reasons for such compensations in much greater detail than the Functional Movement Screening. The practicality of using the overhead squat as a screening method would appear to be quite strong, as it challenges the mobility of all key joints in the kinetic chain through a movement pattern so commonly used in health care practice. There is also the time efficiency benefit of using just one screening assessment in comparison to the Functional Movement Screening' seven. Although the literature has not focused on this particularly, the notion of 'saving time' is always an important reality in the field and a thorough Functional Movement Screening protocol is likely to take around 10 minutes per participates. In recent years, the research looking at the overhead squat would appear to be growing. Atkins, et al. [2] investigated the presence of bilateral imbalance in 105 elite youth soccer players. It has been previously acknowledged that full flexion of the shoulder joint during the overhead squat pattern will challenge the extensibility of the latissimus dorsi muscle [3]. Butler, et al. [4] undertook a biomechanical analysis of the overhead squat assessment in 28 subjects and interpreting the results with 3D motional analysis shows there was no significant difference between the two technique.

\section{Conclusion}

In conclusion, the many research explains a number of areas where the overhead squat may be considered as a method for screening movement quality. Notable differences in Ground reaction forces ${ }^{2}$, joint positions, kinematic interpretation and muscle activation have been depicted, suggesting that multiple considerations do exist for this one screening assessment [4-6]. In future, further studies are needed to utilize the overhead squat assessment as a screening method in varying population, so that the merits and demerits of the overhead squat assessment screening method can be dealt. However, from a practical perspective, not all researchers will have access to expensive force plates, motion analysis systems or electromyography (EMG), and thus, the overhead squat assessment can be viable. 


\section{Journal of Orthopedics \& Bone Disorders}

\section{References}

1. Beardsley C, Contreras B (2014) The Functional Movement Screen: A Review. Strength \& Conditioning Journal 36(5): 72-80.

2. Atkins SJ, Bentley I, Hurst HT, Sinclair JK, Hesketh C (2013) The presence of bilateral imbalance of the lower limbs in elite youth soccer players of different ages. Journal of Strength \& Conditioning Research 30(4): 10071013.

3. Bishop C, Villiere A, Turner A (2016) Addressing movement patterns using the overhead squat. Professional Strength \& Conditioning Journal 40: 712.
4. Butler RJ, Plisky PJ, Southers C, Scoma C, Kiesel KB (2010) Biomechanical analysis of the different classifications of the Functional Movement Screen deep squat test. Sports Biomechanics 9(4): 270-279.

5. Mauntel TC, Post EG, Padua DA, Bell DR (2015) Sex differences during an overhead squat assessment. Journal of Applied Biomechanics 31(4): 244-249.

6. Aspe RR, Swinton PA (2014) Electromyographic and kinetic comparison of the back squat and overhead squat. Journal of Strength \& Conditioning Research 28(10): 2827-2836. 